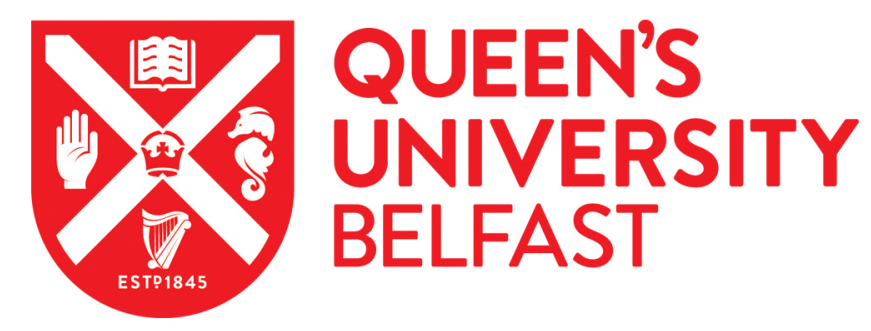

\title{
A Computational Model of Thalamocortical Dysrhythmia in People With Tinnitus
}

Gault, R., McGinnity, T. M., \& Coleman, S. (2018). A Computational Model of Thalamocortical Dysrhythmia in People With Tinnitus. IEEE Transactions on Neural Systems and Rehabilitation Engineering, 26(9), 1845-1857. https://doi.org/10.1109/TNSRE.2018.2863740

Published in:

IEEE Transactions on Neural Systems and Rehabilitation Engineering

Document Version:

Peer reviewed version

Queen's University Belfast - Research Portal:

Link to publication record in Queen's University Belfast Research Portal

Publisher rights

(c) 2018 IEEE.

This work is made available online in accordance with the publisher's policies. Please refer to any applicable terms of use of the publisher.

\section{General rights}

Copyright for the publications made accessible via the Queen's University Belfast Research Portal is retained by the author(s) and / or other copyright owners and it is a condition of accessing these publications that users recognise and abide by the legal requirements associated with these rights.

Take down policy

The Research Portal is Queen's institutional repository that provides access to Queen's research output. Every effort has been made to ensure that content in the Research Portal does not infringe any person's rights, or applicable UK laws. If you discover content in the Research Portal that you believe breaches copyright or violates any law, please contact openaccess@qub.ac.uk. 


\title{
A Computational Model of Thalamocortical Dysrhythmia in People with tinnitus
}

\author{
Richard Gault, Thomas Martin McGinnity, Senior Member, IEEE, and Sonya Coleman, Member, IEEE
}

\begin{abstract}
Tinnitus is a problem that affects a diverse range of people. One common trait amongst people with tinnitus is the presence of hearing loss, which is apparent in over $90 \%$ of the cohort. It is postulated that the remainder of people with tinnitus have hidden hearing loss in the form of cochlear synaptopathy. The loss of hearing sensation is thought to cause a reduction in the bottom-up excitatory signals of the auditory pathway leading to a change in the frequency of thalamocortical oscillations known as Thalamocortical Dysrhythmia (TCD). The downward shift in oscillatory behaviour, characteristic of TCD, has been recorded experimentally but the underlying mechanisms responsible for TCD in tinnitus subjects cannot be directly observed. This paper investigates these underlying mechanisms by creating a biologically faithful model of the auditory periphery and thalamocortical network, called the central auditory processing (CAP) model. The proposed model replicates tinnitus related activity in the presence of hearing loss and hidden hearing loss in the form of cochlear synaptopathy. The results of this work show that both bottom-up and top-down changes are required in the auditory system for tinnitus related hyperactivity to coexist with TCD, contrary to the theoretical model for TCD. The CAP model provides a novel modelling approach to account for tinnitus related activity with and without hearing loss. Moreover, the results provide additional clarity to the understanding of TCD and tinnitus and provide direction for future approaches to treating tinnitus.
\end{abstract}

Index Terms-Tinnitus, thalamocortical dysrhythmia, cochlear synaptopathy, top-down inhibition.

\section{INTRODUCTION}

$\mathbf{T}$ INNITUS is the phantom perception of a sound heard in or around the head in the absence of an external source, affecting $10-15 \%$ of the population, with $1-3 \%$ of people consequently experiencing a diminished quality of life [1]. There are many forms of tinnitus, that are primarily broken down into two groups: objective tinnitus and subjective tinnitus. Objective tinnitus refers to sounds coming from the ear that can be heard by clinicians and are apparent during an examination. These sounds are generated mechanically within the ear and are referred to as somato-sounds. Subjective tinnitus refers to phantom sounds that are only apparent to the person experiencing the phenomena. This paper will only consider subjective tinnitus that will herein be referred to simply as tinnitus.

R. Gault and TM. McGinnity are with the College of Science and Technology, Nottingham Trent University, Nottingham, NG1 4FQ, U.K. e-mail: (richard.gault,martin.mcginnity@ntu.ac.uk).

Sonya Coleman is with the Intelligent Systems Research Centre, University of Ulster, Londonderry, UK. TM. McGinnity is also with the Intelligent Systems Research Centre, University of Ulster, Londonderry BT48 7JL, U.K.e-mail: (sa.coleman,tm.mcginnity@ulster.ac.uk).

Pre-print
Tinnitus is apparent in a wide and diverse range of people and it is therefore important to focus on the similarities that unite the tinnitus cohort. Over $90 \%$ of people with tinnitus have observable hearing loss [2]. It is possible that those with normal hearing thresholds have hidden hearing loss. One form of hidden hearing loss is cochlear synaptopathy. Cochlear synaptopathy occurs when the synaptic terminals between the hair cells of the cochlea and the dendrites of the auditory nerve (AN) fibres are lost forever. The effects of cochlear synaptopathy are variable across those AN fibres with low spontaneous firing rates $(\leq 10 \mathrm{~Hz})$, AN fibres with a medium spontaneous firing rates $(\leq 20 \mathrm{~Hz})$ and AN fibres with a high spontaneous firing rate $(\geq 40 \mathrm{~Hz})$. A bias towards the loss of low and medium spontaneous synaptic terminals has been shown in animal studies [3].

The functional impact of high spontaneous fibre loss is reflected in reduced auditory sensitivity to soft sounds whilst low-and medium-spontaneous fibre loss is reflected by a reduced sensitivity in deafferented frequencies [4]. Tinnitus related studies have shown evidence of cochlear synaptopathy using auditory brainstem response (ABR) [5], [6] (Figure 1). In these studies, wave I amplitudes were reduced in tinnitus subjects; a characteristic of indicative of cochlear synaptopathy [7]. A similar ABR study found no significant differences in the between tinnitus subjects and controls [8]. However, it is noted in [8] that variability of ABRs across subjects and challenges in the recording methodology may obscure subtle evidence for synaptopathy.

The loss of sensory input to the auditory system, caused by hearing damage, is believed to trigger adaptive changes which lead to the amplification of spontaneous activity in regions of hearing loss. Kaltenback et al., [9] found increased activity in the Dorsal Cochlear Nucleus (DCN) of hamsters with behavioural signs of tinnitus. Koehler and Shore [10] showed that spike timing dependent plasticity could be responsible for the auditory systems adaptation to multisensory imbalance following hearing loss. Other approaches consider homeostatic plasticity as a trigger for gain adaptation in the DCN, which regulates the mean firing rate of neurons to ensure they do not become too over or under active [11]. Schaette and McAlpine [5], Kehrle et al., [12] and et al., [6] investigated changes in the auditory periphery measuring the ABR. These studies found changes in the ratio of amplitudes of components I and V of the ABR in tinnitus subjects compared with control subjects. Despite an initially reduced amplitude of the I component, the amplitude of the $\mathrm{V}$ component was comparable to those with no tinnitus indicating an amplification of the signal had occurred [5]. Animal studies 


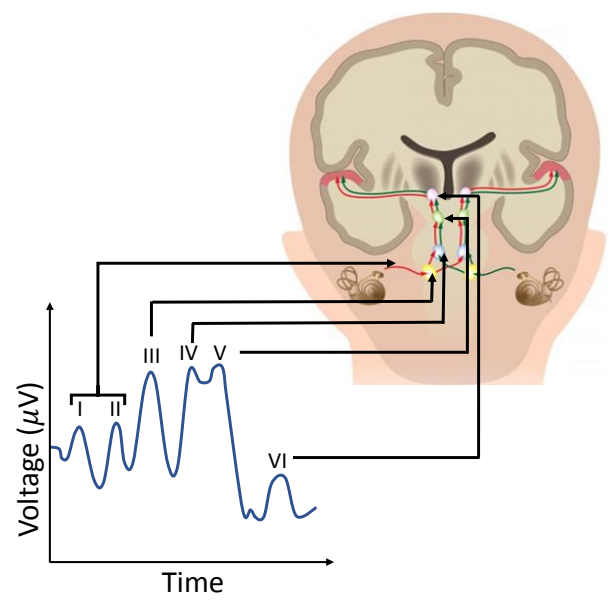

Fig. 1. Illustration of a typical ABR where the peaks or components of the waveform have been mapped to the source of activity [18]

have also shown similar behaviour following noise induced cochlear synaptopathy [13]. It was shown that gain adaptation is a possible mechanism behind the apparent amplification using a computational model. Furthermore, the location for this adaptation within the DCN is in keeping with empirical findings [9], [10]. Latencies in the ABR have also been shown to be a possible marker for cochlear synaptopathy [14]. Other approaches using envelope following response paradigms have shown evidence of cochlear synaptopathy [15].

A controlled approach investigated sound deprivation is to use an ear plug, worn for a sustained period of time, to mimic hearing loss and produce reversible tinnitus [16]. This technique has also been shown to increase central gain governed by adaptation in the brainstem [17]. The location of this adaptation is thought to be the cochlear nucleus [5], [6]. The findings in [6] interpret the ventral cochlear nucleus (VCN) to be the location for the adaptation based on localisation of the ABR while [5] interpret the origin of the adaptation to be the DCN in line with animal studies [9], [10]. The amplified neuronal noise in the auditory system is postulated to be the basis for the phantom sound that is perceived as tinnitus.

The effects of hearing loss can also have an impact on neural correlates of tinnitus. Thalamocortical dysrhythmia (TCD) is characterised by a downward shift in the frequency of the oscillatory behaviour of the thalamo-cortical network brought about by an imbalance of the excitatory and inhibitory signals within the network. The excitatory and inhibitory imbalance behind TCD can be brought about by bottom-up deafferentation (leading to reduced excitatory signals) and/or top-down noise-cancelling deficit (leading to changes in the inhibitory signals) [19]. TCD begins with hyperpolarisation of thalamic cells that leads to low-threshold calcium spike bursts at a lower frequency (theta: $4-7 \mathrm{~Hz}$ ) than normal (alpha activity: $8-12 \mathrm{~Hz}$ ) [20]. Some experimental studies have found, more generally, enhanced slow wave activity including increased delta activity (1-4Hz) [48], [68], [67], [69]. At a cortcal level an associated increase in gamma $(\geq 30 \mathrm{~Hz})$ activity results in theta-gamma coupling [19].

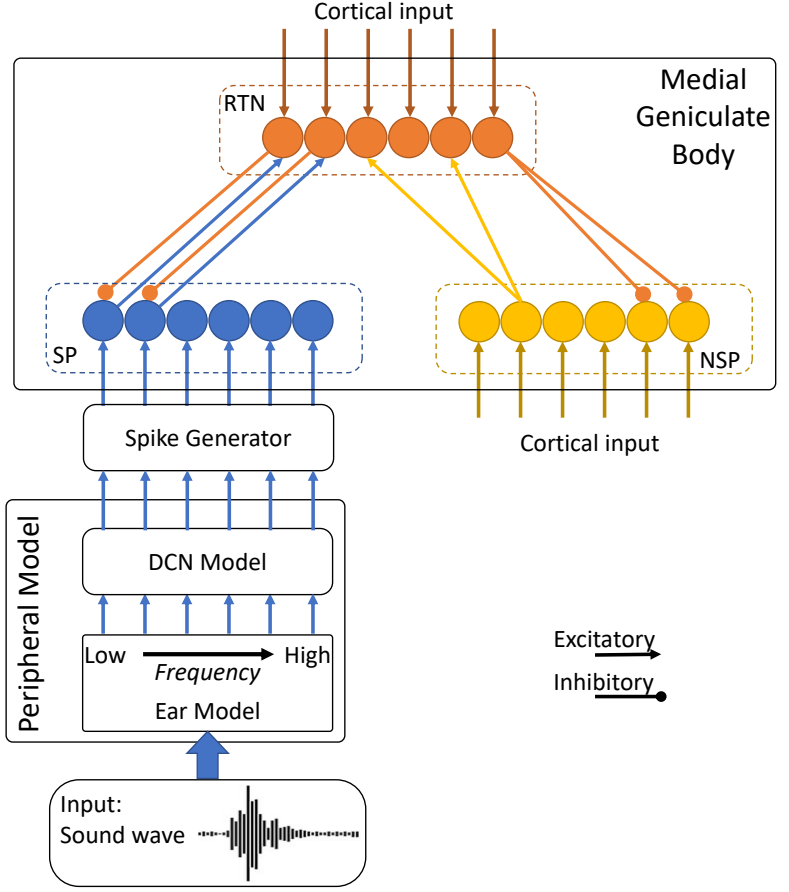

Fig. 2. Outline of the CAP model $[\mathrm{SP}=$ specific projector, NSP $=$ Nonspecific projector and TR $=$ Thalamic Reticular $]$

The oscillatory changes characterising TCD have been observed in electroencephalographic (EEG) and magnetoencephalographic (MEG) studies including tinnitus. [20], [21], [22]. In cases of tinnitus, TCD is postulated to arise from bottom-up changes in the auditory system caused by reduced excitation stemming from hearing loss [20]. Other conditions with a cortical origin that display TCD, such as epilepsy, are thought to be triggered by top-down signals where there is a reduction in the corticothalamic input [20]. In either case, it is over inhibition that causes the dysrhythmia characterised by a slowing of activity from the alpha band to theta band [19]. It is not possible to empirically observe the underlying mechanisms of TCD using non-invasive techniques. Therefore, we propose a computational approach to investigate these mechanisms.

Section II will outline the development of the novel central auditory processing (CAP) model (Figure 2). The CAP model includes the creation of a biologically inspired representation of the auditory periphery, called the peripheral model, that simulates the development of tinnitus related hyperactivity in the auditory brainstem (Section II-A). The peripheral model facilitates a novel approach to modelling cochlear synaptopathy. The CAP model is completed by extending the peripheral model to incorporate the thalamocortical network thus enabling the investigation of tinnitus and TCD (Section II-B). Section III gives an overview of the results before a discussion of the papers findings and its conclusion in Sections IV and $\mathrm{V}$.

\section{Methods}

Modelling hidden hearing loss was first investigated in [5] and further modelling efforts [13] have been made to 


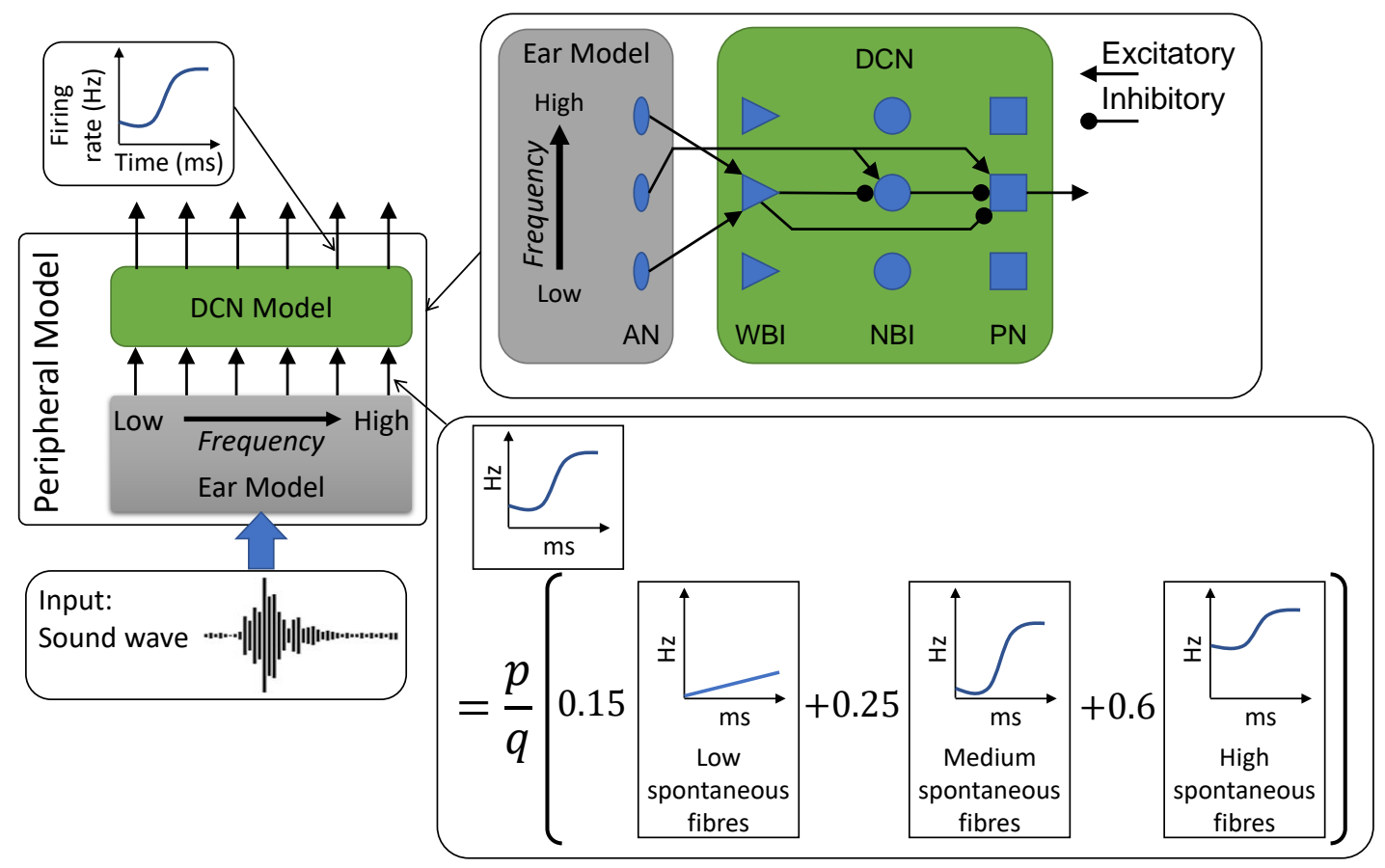

Fig. 3. Topology of the novel peripheral model $[\mathrm{WBI}=$ wideband inhibitor, NBI $=$ Narrowband inhibitor and PN $=$ projector neurons $]$

capture the selective bias to the loss of low spontaneous AN fibres as found experimentally [3]. Our novel approach to this problem of modelling cochlear synaptopathy will aim to describe the broad dynamic range of AN fibres in detail and capture the effect synaptopathy has on low, medium and high spontaneous fibres. The Schaette and Kempter tinnitus model [23] (originally detailed in [24]) will be extended to include a detailed ear model [32] which can capture traditional hearing loss and facilitates the investigation of cochlear synaptopathy.

\section{A. Development of peripheral model}

Figure 3 provides an illustration of the structure of the proposed peripheral model. The peripheral model begins with a biologically inspired ear model whose input is an instantaneous pressure waveform and the output is the firing rate over time of a single AN fibre with a given characteristic frequency from which the mean rate for each characteristic frequency can be calculated. This model has been extensively developed over a number of works [25], [26], [27], [28], [29], [30], [31], [32]. A detailed explanation of the model's behaviour used in this work is given in [32] and is benchmarked against empirical data in previous studies cited above. The ear model depicts low, medium and high spontaneous AN fibres. The tonotopic map is modelled logarithmically between $250 \mathrm{~Hz}$ to $16000 \mathrm{~Hz}$ in steps of $1 / 10$ of an octave to capture the typical frequency range of an adult. As the tonotopic mapping is preserved from the cochlea through to the auditory cortex, there is a canonical one-to-one mapping of characteristic frequencies between each module of the peripheral model (Figure 3).

The number of AN fibres per characteristic frequency in the tonotopic map is inferred using the work of Greenwood
[33] to determine the proportion of the 30000 AN fibres that would be found at each characteristic frequency in humans. The proportion of low, medium and high spontaneous fibres is taken to be $15 \%, 25 \%$ and $60 \%$ respectively and it is assumed that this distribution is uniform across frequencies [34]. Weighted by the proportion of each fibre type and the number of AN fibres per frequency, the AN firing rates are summed into a single mean firing rate over time for each characteristic frequency. The overall net activity, $f_{c}$, of the AN fibres at a particular frequency, $c$, is modelled by

$$
f_{c}=\frac{p}{q}\left[\begin{array}{lll}
0.6 & 0.25 & 0.15
\end{array}\right]\left[\begin{array}{lll}
h & m & l
\end{array}\right]^{T}
$$

where $p$ is the number of AN fibres that contribute to the net activity of the AN at a given frequency and $q$ is the total number of fibres per characteristic frequency. The firing rates of the low, medium and high spontaneous fibres are denoted as $l, m$ and $h$ respectively. In the case of a healthy ear, $p=q$ and therefore the scalars $p$ and $q$ have negligible impact in Equation 1. For convenience, we use the notation $f_{c}$ rather than $f_{c}(t)$. Figure 4 shows that the behaviour of the net AN activity presented in this work and the AN model by Schaette and Kempter [23]. Whilst neither model is a true reflection of the real world behaviour, both models show a similar trend. The AN model used in [23] is a simplified firing rate model dependent on sound intensity whereas the AN model used in this work is derived from empirical data and biologically plausible distribution of AN fibres. The AN fibres provide input to the DCN module.

The DCN module consists of three populations of neurons; namely wideband inhibitor (WBI), narrowband inhibitor (NBI) and projector neurons (PNs) [35]. The individual neurons are modelled as in the Schaette and Kempter tinnitus model [23] 


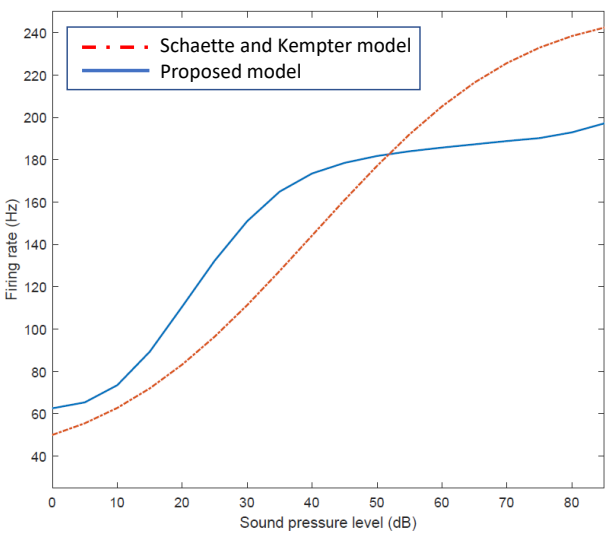

Fig. 4. Model of net AN activity with respect to increasing sound level compared with Schaette and Kempter AN model

and the parameter values are consistent with this study. This particular work will consider only the spontaneous activity in the auditory pathway. Due to an activation threshold of $27 \mathrm{~dB}$ the WBI and NBI neurons will not contribute to the activity of the DCN which will become apparent in Equations 2 and 3. They are detailed here and included in the overall model to facilitate future development and to allow the investigation of the effects sound stimulus may have on a biologically inspired DCN model.

The firing rate AN model from the Schaette and Kempter model is required for the homeostatic calculations presented in [23]. The use of a different AN model in this work, which provides a more biologically faithful AN model, leads to a reformulation of those stages of the Schaette and Kempter DCN model whilst maintaining the same overall behaviour of the original model. The firing rate, $f_{c}$, of the AN fibre for a given characteristic frequency, $c$, provides excitatory input to the WBI, NBI neurons and PNs. The firing rate, $w_{c}$, of a WBI neuron of characteristic frequency $c$ is

$$
\begin{aligned}
w_{c} & =W\left(f_{A}, \ldots, f_{B}\right) \\
& =\left[\frac{1}{N} \sum_{\substack{i=A \\
i \neq c}}^{B} f_{i}-\theta_{i}^{w}\right]_{+}
\end{aligned}
$$

Here frequencies $A, B$ and $c$ satisfy that $c \in\{A, \ldots, B\}$ and $|\{A, \ldots, B\} \backslash\{c\}|=N=10 \%$ of the total size of the tonotopic map. As a result, frequencies $A$ and $B$ can be consider the lower and upper limits of a moving window across the entire tonotopic map. In this work the tonotopic map is represented from $250 \mathrm{~Hz}$ to $8000 \mathrm{~Hz}$ in the case of cochlear synaptopathy and $250 \mathrm{~Hz}$ to $16000 \mathrm{~Hz}$ in the case of hearing loss. Further details of the tonotopic maps will be given in Section II-C. The positive rectifier is denoted by $[\ldots]_{+}$and defined by $[x]_{+}=\max (0, x)$. The notation in Equation 2 is the more generalised version of the original, and equivalent description, given in [23]. It should be noted that there is a sliding window on the input AN activity so that each WBI neuron receives input from adjacent frequencies. The firing threshold of the WBI neurons, $\theta_{i}^{w}$, is chosen such that $f_{i}-\theta_{i}^{w}=0$ when frequency $i$ is stimulated by a sound of $27 \mathrm{~dB}$. Therefore WBI

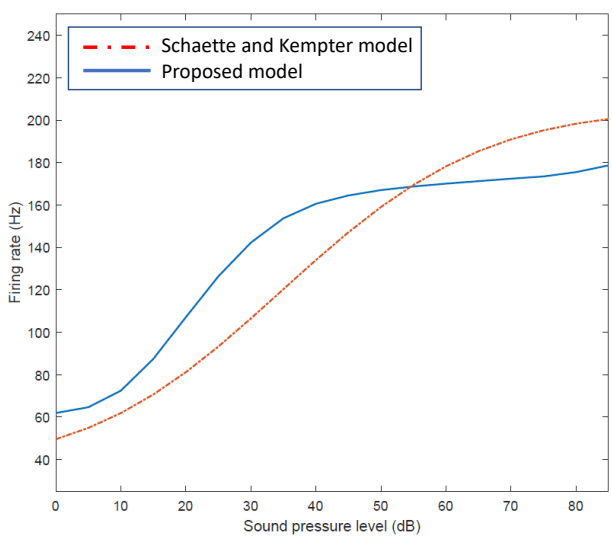

Fig. 5. Model of the PN activity with respect to increasing sound level compared with Schaette and Kempter PN model

neurons are inactive until there is broadband noise of greater than $27 \mathrm{~dB}$. The choice in threshold means that WBI neurons will not be unnecessarily stimulated by spontaneous activity or quiet environments whilst the threshold remains low enough to allow for low level sounds, such as a whisper $(\approx 30 \mathrm{~dB})$, to be processed [23].

The NBI neurons are stimulated by pure tones and are inhibited when there is broadband noise. The firing rate, $n_{c}$, of the NBI neuron with characteristic frequency $c$ is given by

$$
n_{c}=N\left(f_{c}, w_{c}\right)=\left[g_{f} f_{c}-g_{n w} w_{c}-\theta_{c}^{n}\right]_{+}
$$

where the synaptic weight between the WBI neurons and NBI neurons, $g_{n w}=1.5$ (as in [23]), ensures that NBI neurons do not respond to broadband noise. The gain factor for the excitatory AN input is $g_{f}=1$. The firing threshold $\theta_{c}^{n}$ is equal to $\theta_{c}^{w}$ (Equation 2) so that the NBI neurons respond to pure tones in the same way as WBI neurons respond to broadband noise.

The PNs are the final layer in the DCN module within the peripheral model. They receive input from the AN fibres, WBI and NBI neurons. The firing rate of a PN, $r_{c}$, is calculated by

$$
\begin{aligned}
r_{c} & =R\left(f_{c}, w_{c}, n_{c}\right) \\
& =r_{h i g h} \tanh \left(\frac{\left[g_{f} f_{c}-g_{w} w_{c}-g_{n} n_{c}\right]_{+}}{r_{h i g h}}\right)
\end{aligned}
$$

where $r_{\text {high }}=300$ (consistent with [23]) is the maximum possible firing rate of the PNs. The behaviour of the PNs is shown in Figure 5. The values of the gain factors $g_{w}$ and $g_{n}$ can vary the dynamics of the neuron model as explored in [23]. It should be noted that any differences between the AN models of this work and [23] are mapped in the canonical way by the DCN model to the PNs. Consequently, the peripheral model and [23] are analogous and equivalent in terms of behaviour and function. It is within the DCN model that homeostatic plasticity is modelled to adapt the gain factors in Equation 4.

After hearing damage, resulting from cochlear synaptopathy or sensorineural hearing loss, the AN fibres will decrease in their spontaneous and maximum firing rates. Consequently, the input to the PNs is decreased lowering the mean firing 
rate of these neurons. The mean firing rate of the PN with characteristic frequency $c$ is calculated as

$$
\overline{r_{c}}=\frac{1}{f_{\max }^{(c)}-f_{s p}^{(c)}} \int_{f_{c}=f_{s p}^{(c)}}^{f_{\max }^{(c)}} R\left(f_{c}, w_{c}, n_{c}\right) d f
$$

where $f_{s p}^{(c)}$ and $f_{\text {max }}^{(c)}$ are the spontaneous and maximum firing rates of the AN fibres over time. These values are obtained by stimulating the AN fibre with a pure tone centred at the characteristic frequency of the $\mathrm{AN}$ fibre at $0 \mathrm{~dB}$ for spontaneous firing rate and $85 \mathrm{~dB}$ for maximum firing rate. It would not be practical or ethical to validate this model's behaviour through electrophysiological studies which would stimulate the ear at very high sound intensity, for example, $120 \mathrm{~dB}$, and therefore a sound level of $85 \mathrm{~dB}$ is used to calculate $f_{\max }^{(c)}$. It should be noted that WBI and NBI neurons are described as functions of the auditory nerve activity $f_{c}$. Therefore when there is damage to the ear, there will be a reduction in $f_{s p}^{(c)}$ and $f_{\max }^{(c)}$ and consequently a reduction in the activity of the WBI and NBI neurons as well as the PNs.

In order to adapt to the reduced mean firing rate the gain within the PN model is adjusted. By altering Equation 4, the gain factor $g \in[1,3]$ aims to stabilise the mean firing rate such that the firing rate of a PN can now be written as

$$
\begin{aligned}
r_{c} & =R\left(f_{c}, w_{c}, n_{c}\right) \\
& =r_{h i g h} \tanh \left(\frac{\left[g f_{c}-\frac{g_{w}}{g} w_{c}-\frac{g_{n}}{g} n_{c}\right]_{+}}{r_{h i g h}}\right) .
\end{aligned}
$$

The value of $g$ is calculated numerically. The firing rates from the PN layer provide the input to the thalamocortical model.

\section{B. Development of the central auditory processing (CAP) model}

The peripheral model is extended to include the thalamocortical network as previously illustrated in Figure 2. The thalamocortical system is modelled by three populations of neurons [36]; specific projector (SP) neurons, non-specific projector (NSP) neurons and thalamic reticular (TR) neurons. The SP neurons receive excitatory input from the auditory periphery and collectively represent the Medial Geniculate Body (MGB). Although projections do exist directly from the DCN to the MGB [37], most of the input for the MGB comes from the inferior colliculus (IC). Manzoor et al., [38] showed that the IC is a passive component to tinnitus related activity. Moreover, hyperactivity in the IC is proportional to the activity in the DCN and thus acts as a passive relay for tinnitus related hyperactivity [39]. In both studies it was possible to categorically conclude that the IC inherited the hyperactivity from the DCN keeping activity consistent along the tonotopic mapping between the two regions of the brainstem. Therefore, without loss of generality, we omit this region from the proposed model. The TR neurons receive excitatory input from the cortex, SP and NSP populations and project inhibitory signals towards the SP and NSP populations. The TR and SP networks are connected in a bijective fashion whilst the TR and NSP networks are connected in a one-to-many fashion in both directions. A single neuron in either the TR or NSP network projects to $15 \%$ of its codomain. Although these connections are random we restrict the span of each neuron's connections to exist within a window size of $20 \%$ of the total number of neurons. This restriction is introduced to ensure some degree of consistency between executions of the simulation.

Each neuron in the SP network corresponds to a characteristic frequency in the auditory periphery and every network within the thalamocortical model has the same number of neurons. The input for each thalamic neuron is a Poissondistributed spike train. The NSP and TR networks receive excitatory input from the cortex. The top-down cortical input is known to be at a rate $\leq 100$ spikes per second [40]. To avoid the extreme values of this cortical input, the input to the TR and NSP neurons consists of pseudo-random Poisson distributed spike trains between $50-60 \mathrm{~Hz}$. The SP network receives input from the auditory periphery model in the form of a Poisson distributed spike train with a spike rate corresponding to the output of their associated PNs. The output spikes of every neuron in the thalamocortical network are binned into $10 \mathrm{~ms}$ time segments and a moving average is used to smooth the binned data with a sliding window of size 5 [36]. A fast Fourier transform is then applied to the smoothed data to map the time series data to the frequency domain. This process was repeated 10 times and the power spectra of the 10 simulations are averaged to improve the signal to noise ratio. To evaluate the existence of TCD the dominant frequency of oscillation is calculated for the control, hearing loss and cochlear synaptopathy scenarios.

To model the thalamic neurons a conductance based leaky integrate-and-fire-or-burst neuron is used [41]. The change in membrane potential over time is described by

$$
\frac{d V}{d t}=\frac{1}{C}\left(I_{i n p}-I_{L}-I_{T}\right)
$$

where $C$ is the capacitance per unit area. The input current $I_{\text {inp }}$ is defined as

$$
I_{i n p}=\sum_{i} g_{s_{i}}\left(E_{r e v}-V\right)
$$

The input current outlined in [41] corresponds to a single neuron whereas the input current modelled here (and equivalently in [36]) is associated with inputs from other neurons within the network. The reversal potentials, $E_{\text {rev }}$, are separated into excitatory and inhibitory synapses, which are individually denoted $E_{\text {exc }}$ and $E_{i n h}$ respectively, and synaptic conductance is denoted $g_{s}$. The post synaptic conductances, $g_{s}$ are modelled by the decay function

$$
\frac{d g_{s}}{d t}=-\frac{g_{s}}{\tau_{s}}+\sum \delta(t-d) .
$$

The maximum conductances $\left(g_{s}\right)$, signal delays $(d)$ and time constants $\left(\tau_{s}\right)$, associated with each connection are given in Table II.

The leakage current, $I_{L}$, is given by

$$
I_{L}=g_{L}\left(V-E_{L}\right)
$$

where $g_{L}$ is the conductance associated with the leakage current and $E_{L}$ is the reversal potential. The $t$-current, $I_{T}$, 
TABLE I

VALUES OF GENERIC PARAMETERS FOR THE THALAMOCORTICAL MODEL.

\begin{tabular}{lll}
\hline Parameter & Unit & Value \\
\hline$V_{\theta}$ & $m V$ & -35 \\
$V_{\text {spike }}$ & $m V$ & 30 \\
$V_{\text {reset }}$ & $m V$ & -50 \\
$g_{L}$ & $m S / \mathrm{ms}^{2} / \mathrm{cm}^{2}$ & 0.035 \\
$E_{L}$ & $m V$ & -65 \\
$g_{T}$ & $m V$ & 0.07 \\
$\tau_{h_{2}}$ & $m s$ & 100 \\
$E_{T}$ & $m V$ & 120 \\
$C$ & $\mu F / \mathrm{cm}^{2}$ & 2 \\
$E_{\text {exc }}$ & $m V$ & 0 \\
$E_{\text {inh }}$ & $m V$ & -85 \\
$\tau_{s}$ (excitatory) & $m s$ & 20 \\
$\tau_{s}$ (inhibitory) & $m s$ & 30 \\
\hline
\end{tabular}

represents the ionic current flow resulting from $t$-channel activation. Low voltage activates these channels leading to calcium bursts upon depolarisation off the cell. The $t$-current is modelled as

$$
I_{T}=g_{T} m_{\infty} h\left(V-E_{T}\right)
$$

where $g_{T}$ is the conductance, $E_{T}$ is the reversal potential and $m_{\infty}=\frac{d}{d x} \max \{x, 0\}$, i.e., the Heaviside step function. The inactivation variable, $h$, is modelled by the decay function

$$
\frac{d h}{d t}= \begin{cases}\frac{-h}{\tau_{h_{1}}}, & \text { if } V \geq V_{h} \\ \frac{1-h}{\tau_{h_{2}}}, & \text { otherwise. }\end{cases}
$$

If $V \geq V_{h}$, bursting activity will be present and thus $\tau_{h_{1}}$ will control the length of the burst. For neurons in the SP and NSP networks $\tau_{h_{1}}=20 \mathrm{~ms}$ and for neurons in the TR network $\tau_{h_{1}}=40 \mathrm{~ms}$. Conversely if $V<V_{h}$, the inactivation tends to 1. Therefore the time constant $\tau_{h_{2}}$ controls the rate in which the thalamic neuron de-inactivates so that calcium channels are in a state that facilitates bursting activity to occur. The value of $\tau_{h_{2}}$ is $100 \mathrm{~ms}$ for all three networks. In [41], $V_{h}=-60 \mathrm{mV}$ as a single neuron whereas in this work (and equivalently in [36]) $V_{h}$ is -64 for neurons of the TR network and -66 for neurons of the NSP and SP networks. The value of $V_{h}$ differs in the TR network compared with the NSP and SP networks to account for known morphological differences between the neuron groups leading to distinctly different behaviour [42].

The parameters used during implementation are given in Tables I and II. The parameters outlined in Table I and the majority of parameters in Table II are identical to those given in [36]. There are differences between the implementation of this paper and [36]; namely the network size and the peripheral input firing rates to each network. The cortical input firing rates given in [36] are much higher than the $100 \mathrm{~Hz}$ found in reality [40]. So that the model's synchronous activity is predominantly in the alpha band when at rest the connectivity weights between neurons have been adjusted with no adjustments made to parameters associated with the biological mechanisms of the thalamocortical neurons; such as the resting potential, spiking threshold or decay rates.

\section{Modelling cochlear synaptopathy}

The ear model used within the peripheral model already models hearing loss as recorded from an audiogram. Normal
TABLE II

VALUES OF POPULATION RELATED PARAMETERS FOR THE THALAMOCORTICAL MODEL ( $\mathrm{P}=$ PERIPHERY,CRX=CORTEX).

\begin{tabular}{lll}
\hline Parameter & Connection & Value \\
\hline$g_{s}(m S)$ & $\mathrm{P} \rightarrow \mathrm{SP} / \mathrm{NSP}$ & 0.005 \\
& $\mathrm{CRX} \rightarrow \mathrm{TR}$ & 0.01 \\
& $\mathrm{SP} \rightarrow \mathrm{TR}$ & 0.02 \\
& $\mathrm{NSP} \rightarrow \mathrm{TR}$ & 0.01 \\
& $\mathrm{TR} \rightarrow \mathrm{SP}$ & 0.0025 \\
& $\mathrm{TR} \rightarrow \mathrm{NSP}$ & 0.00375 \\
$\tau_{s}(m s)$ & $\mathrm{P} \rightarrow \mathrm{SP} / \mathrm{NSP}$ & 7 \\
& $\mathrm{CRX} \rightarrow \mathrm{TR}$ & 10 \\
& $\mathrm{SP} \rightarrow \mathrm{TR}$ & 20 \\
& $\mathrm{NSP} \rightarrow \mathrm{TR}$ & 20 \\
& $\mathrm{TR} \rightarrow \mathrm{SP}$ & 30 \\
& $\mathrm{TR} \rightarrow \mathrm{NSP}$ & 30 \\
Delay $(m s)$ & $\mathrm{P} \rightarrow \mathrm{SP} / \mathrm{NSP}$ & 0 \\
& $\mathrm{CRX} \rightarrow \mathrm{TR}$ & 7 \\
& $\mathrm{SP} \leftrightarrow \mathrm{TR}$ & 3 \\
& $\mathrm{NSP} \leftrightarrow \mathrm{TR}$ & 3 \\
$V_{h}(m V)$ & $\mathrm{TR}$ & -64 \\
& $\mathrm{SP} / \mathrm{NSP}$ & -66 \\
$\tau_{h_{1}}(m s)$ & $\mathrm{TR}$ & 40 \\
& $\mathrm{SP} / \mathrm{NSP}$ & 20 \\
\hline
\end{tabular}

hearing thresholds are only defined up to $8000 \mathrm{~Hz}$ specifying a hearing threshold of no more than $20 \mathrm{~dB}$ for each frequency. Consequently, when modelling cochlear synaptopathy the tonotopic map will incorporate frequencies from $250 \mathrm{~Hz}$ to $8000 \mathrm{~Hz}$ in steps of $1 / 10$ of an octave even though the hearing loss case considers frequencies up to $16000 \mathrm{~Hz}$. The AN activity for a healthy ear with no damage is calculated for all frequencies as well as the AN activity of an ear on the borderline of normal hearing thresholds; that is $20 \mathrm{~dB}$ hearing threshold at all frequencies. Hence the AN activity following a hearing threshold of $20 \mathrm{~dB}$ is determined for all frequencies. The dendrites of the AN fibres have a single synaptic terminal with a single inner hair cell without branching [43] so it suffices to model the loss of a synaptic terminal by removing the corresponding AN fibre from the overall net activity.

Although the exact ratio in which the different AN fibre types are lost is not known, it has been found that low spontaneous fibres are more susceptible to synaptic loss than high spontaneous fibres [3]. Moreover, [3] found that up to $30 \%$ of all terminals could be lost with a fractional loss of low and medium spontaneous fibres of $38 \%$. Similar studies have found that up to $50 \%$ of the total terminals can be lost at particular frequencies whilst normal hearing levels are maintained [44]. An optimisation problem can be constructed to identify feasible values for the ratio in which high, medium and low spontaneous fibres are lost such that the error between the modelled synaptic loss and the empirical results from [3] is minimised. In this work, a Genetic Algorithm (GA) was used to find solutions to this optimisation problem and was implemented in Matlab R2013a (The MathWorks, Natick, MA, USA) using the Global Optimization Toolbox. The GA terminated with a minimal solution, i.e. the error was zero, and found that the ratio of high, medium and low spontaneous fibre loss is $3: 25: 34$. That is, for every 3 high spontaneous fibres lost there are 25 medium spontaneous fibres and 34 low spontaneous fibres lost. Through an iterative process the synaptic terminals were deleted in this ratio for each 
a)

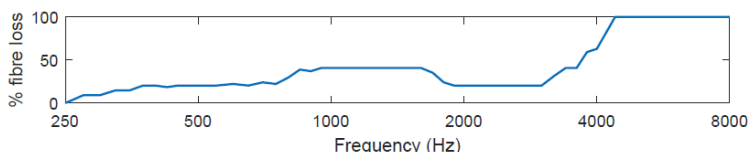

b)

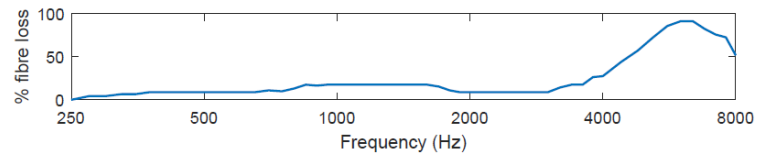

c)

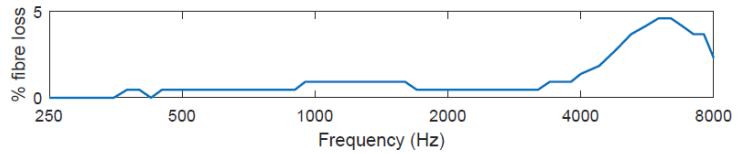

d)

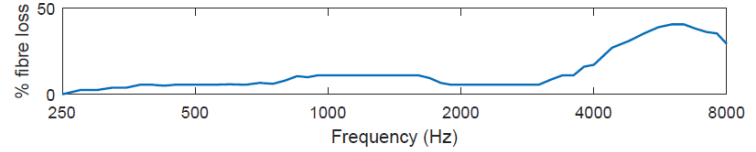

Fig. 6. The percentage of AN fibres lost through cochlear synaptopathy (a) low spontaneous fibres, (b) medium spontaneous fibres, (c) high spontaneous fibres, (d) all fibres

characteristic frequency until the net AN fibre activity was on the limit of AN activity of someone with the minimum thresholds for normal hearing.

Following cochlear synaptopathy the proposed model showed a total loss of up to $41 \%$ with a fractional loss of $38 \%$ in low and medium spontaneous fibres in line with empirical results [3], [44]. Less than 5\% of all high spontaneous fibres were lost, consistent with the empirical results that found no significant difference between the high spontaneous terminal count with and without damage [3]. Figure 6 shows the percentage loss of synaptic terminals across all frequencies for each fibre type whilst maintaining normal hearing thresholds. It should be noted that the $y$-axis in each graph is scaled relative to the overall $\%$ loss in each case and is therefore different for each case. Analytical analysis of the findings presented in [3] has been explored in [4].

\section{RESULTS}

\section{A. Modelling tinnitus related hyperactivity}

The first stage of the investigation requires the analysis of the peripheral model and the evaluation of its ability to model tinnitus related activity in the auditory brainstem. This involves the simulation of hearing loss and cochlear synaptopathy to induce homeostatic changes in the DCN.

Hearing loss was modelled based on high frequency hearing loss, typically found in people with tinnitus, as illustrated in the audiogram shown in Figure 7. The inner and outer hair cell parameters were tuned for the ear model to account for this hearing loss using an audiogram fitting function available with the open sourced ear model [45]. Stimulus for the ear model is sampled at $100 \mathrm{kHz}$ and averaged over 50 repetitions to avoid irregularities. Each stimulus lasts for $350 \mathrm{~ms}$ with a rise and fall ramp of $2.5 \mathrm{~ms}$. For the purposes of stationary analysis (i.e. ignoring the temporal aspect of the model) the mean AN firing rate for each characteristic frequency was calculated between $200 \mathrm{~ms}$ to $300 \mathrm{~ms}$ from stimulus onset. The induced hearing

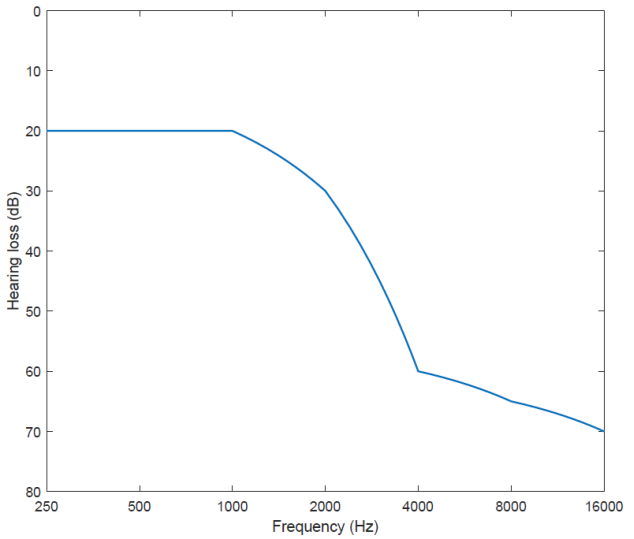

Fig. 7. Example of simulated audiogram showing high frequency hearing loss.

loss leads to a reduction in both $f_{s p}$ and $f_{\max }$ compared with healthy conditions (Figure 8).

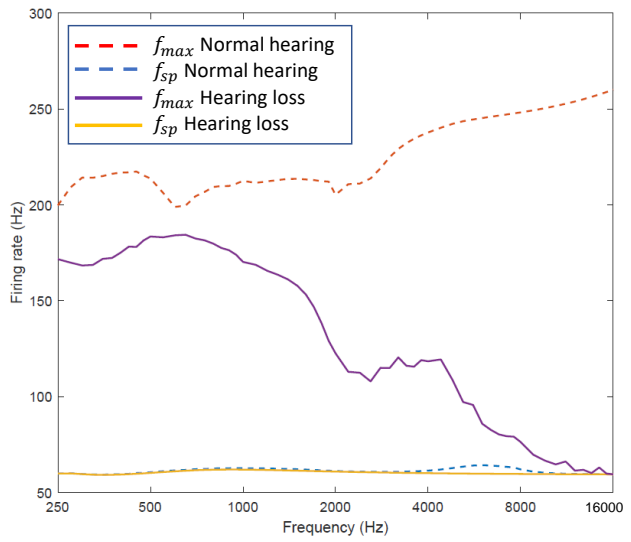

Fig. 8. Decreased $f_{s p}$ and $f_{\max }$ in regions of hearing loss

The spontaneous firing rate of the PNs at the final stage of the DCN is shown in Figure 9 for both the hearing loss and healthy control cases.

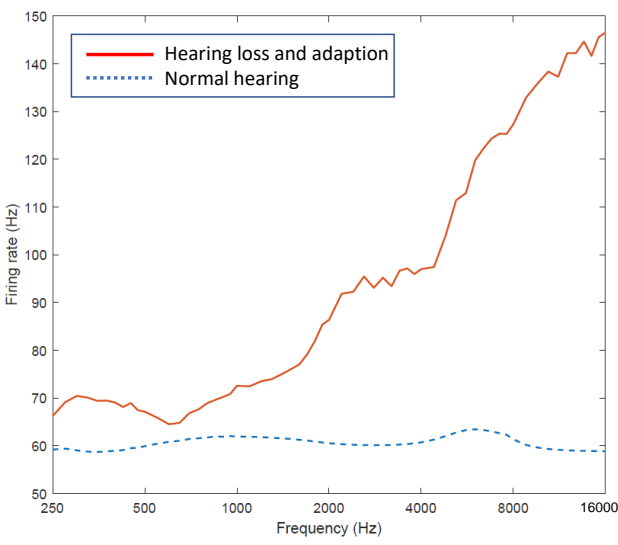

Fig. 9. Elevated activity following hearing loss and gain adaptation.

There is elevated spiking activity in frequencies where hearing loss is simulated as found in cases of tinnitus with 
hyperactivity [9]. The firing rate of the $\mathrm{PN}$ is higher than similar modelling work which used comparable hearing thresholds [11]. However, the resultant behaviour is equivalent to the findings of [11] as Figure 5 clearly shows that the activity of PNs, in both this work and [11], reflect that of sound stimulus at approximately $25-30 \mathrm{~dB}$ in the DCN. A paired t-test found that the overall spontaneous activity across all frequencies following gain adaptation was significantly different $(p<0.01)$ than the control case.

When hearing damage is caused by cochlear synaptopathy, the ratio of high, medium and low spontaneous fibre loss of 3:25:34 was used. Synaptic terminal loss was modelled up to the maximum limits of normal hearing. Similar to the hearing loss case, the reduced activity to the net AN firing rate leads to reduced input to the DCN module (Figure 10).

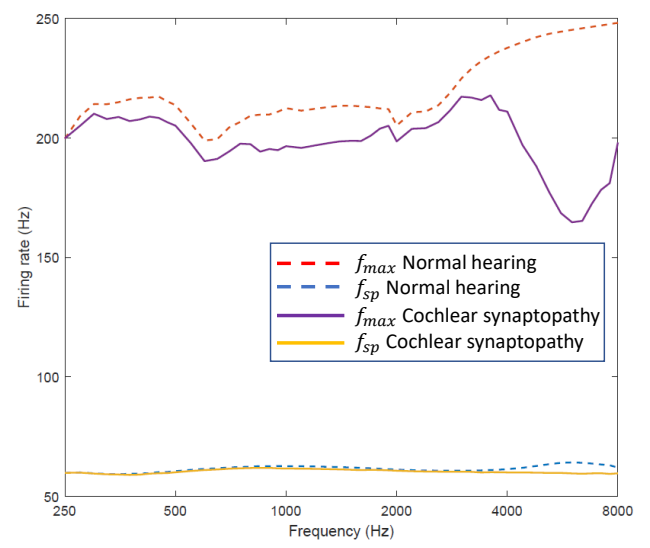

Fig. 10. Decreased $f_{s p}$ and $f_{\max }$ following cochlear synaptopathy

At the DCN level, a paired t-test determined that there was a significant difference between the increased spontaneous activity, or hyperactivity, of the PNs (illustrated in Figure 11) and the spontaneous activity of the healthy control case $(p<0.01)$. The greatest elevation in PN activity is in regions of the tonotopic map with the most synaptopathy (Figures 6 and 11). As in other computational models of tinnitus we

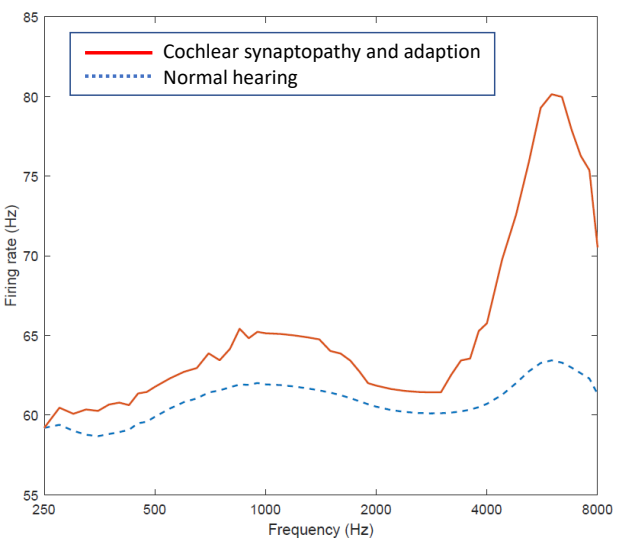

Fig. 11. The increase in synaptic gain causes an increase in the spontaneous firing rate of the PNs following cochlear synaptopathy

postulate that the significant hyperactivity in regions of hearing loss would lead to a phantom noise at a perceptual level.

\section{B. Thalamocortical network with only bottom-up adaptation}

The power spectra of the oscillatory activity of the thalamocortical network for the hearing loss and cochlear synaptopathy related tinnitus scenarios along with their corresponding control cases are shown in Figure 12. The dominant frequencies of oscillation were $9.6 \mathrm{~Hz}$ for the hearing loss scenario and $9.2 \mathrm{~Hz}$ for the corresponding control case. In the case of cochlear synaptopathy and the corresponding control case the dominant frequencies were $9.8 \mathrm{~Hz}$ and $9.3 \mathrm{~Hz}$ respectively. The small difference between the control case for hearing loss and the control case for cochlear synaptopathy is due to the size of the networks being different because of a smaller tonotopic map modelled in the cochlear synaptopathy scenario. A paired t-test was used to compare the power spectra of the control case with the corresponding tinnitus case. In each scenario there was no significant change in the power spectra $(p>0.05)$.

With no apparent decrease in oscillatory activity the natural progression is to consider if the oscillatory changes in tinnitus related TCD are caused by over-inhibition stemming from an increase in top-down inhibition. Consequently, we now investigate the effect increasing the intrinsic inhibition of the thalamocortical network has on the oscillatory behaviour of the thalamic neurons.

Figure 13 shows the effect an increase in inhibition has on the dominant frequency of oscillation for the thalamocortical network under 'healthy' (i.e no hearing loss) conditions. This is determined by subjecting the control case to a multiplicative increase of the gain factor, $g_{s}$, by a scalar $\lambda$ for the TR $\rightarrow$ NSP and SP connections. That is, Equation 8 for the SP and NSP is now rewritten as

$$
I_{i n p}=\sum_{i_{E}} g_{s}\left(V-E_{e x c}\right)+\sum_{i_{I}} \lambda g_{s}\left(V-E_{i n h}\right)
$$

where $i_{E}$ and $i_{I}$ index the excitatory and inhibitory presynaptic connections respectively. The conclusion from the previous section along with this result indicates that although dysrhythmia is not apparent in tinnitus subjects because of peripheral damage, the internal gain adaptation on the inhibitory weights can cause the slowing of dominant oscillatory behaviour. Figures $14 \mathrm{a}$ and $14 \mathrm{~b}$ illustrate the normalised power spectrum of the thalamocortical network after inhibitory adaptation. The minimal multiplicative scalars required to generate a shift in oscillatory behaviour from alpha to theta were $\lambda=2.4$ and $\lambda=2.3$ for the hearing loss and cochlear synaptopathy cases respectively. With an increase in top-down inhibition, the dominant frequency of oscillation is located in the theta band in both tinnitus cases whilst the healthy control cases predominantly display oscillatory activity in the alpha band. This is characteristic of TCD as observed in EEG and MEG studies [22], [46], [47], [49].

\section{Discussion}

Tinnitus is thought to be generated by maladaptation within the auditory system following hearing loss. Although most people with tinnitus have some form of hearing loss, tinnitus is also observed in subjects with apparently normal hearing 


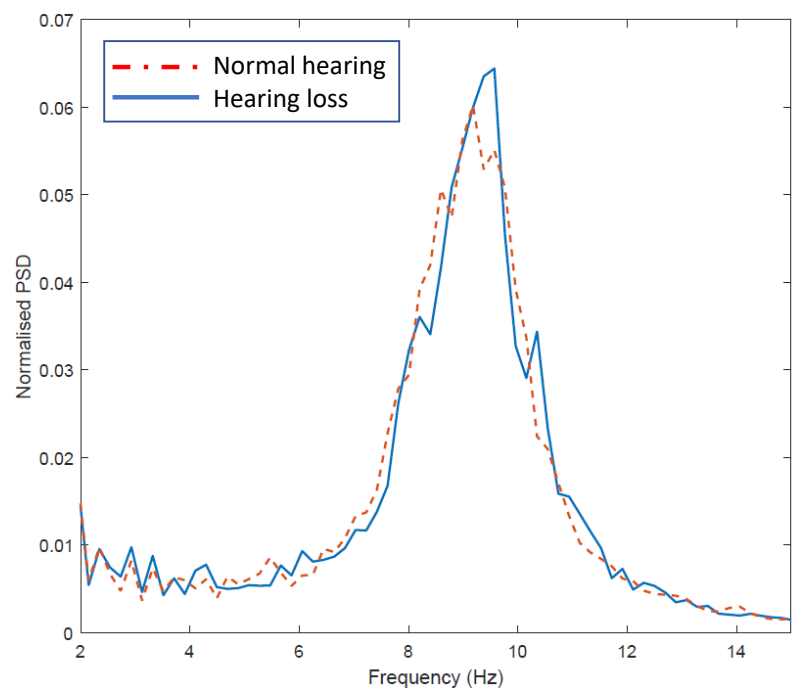

(a) Power spectrum of thalamocortical network with hearing loss and tinnitus

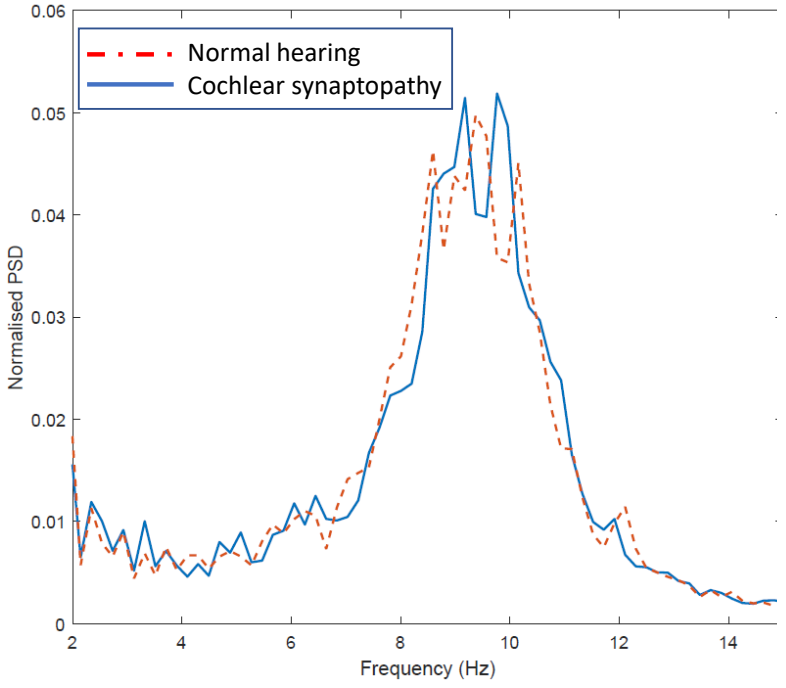

(b) Power spectrum of thalamocortical network with cochlear synaptopathy and tinnitus

Fig. 12. Normalised power spectrum the thalamocortical network in cases of tinnitus

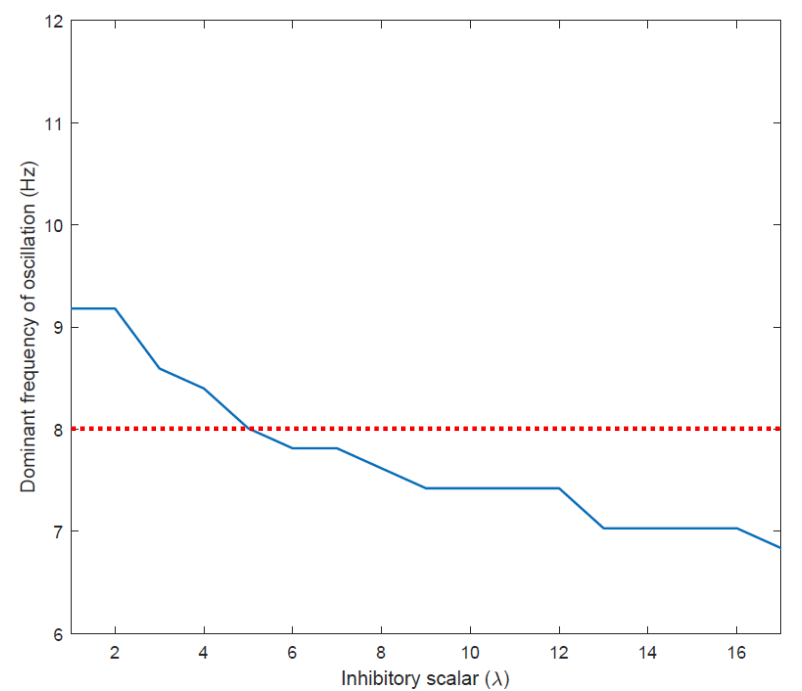

Fig. 13. The dominant frequency of oscillation of the thalamocortical network as function of the strength of the inhibitory connections. The dotted red line $(8 \mathrm{~Hz})$ marks the lower bound of the alpha band.

thresholds. Animal studies [44] and in vivo electrophysiological recordings for humans [7] indicate that significant damage can occur within the ear even when normal hearing is present. Cochlear synaptopathy has been proposed one possible form of hidden hearing loss. The proposed peripheral model shows that cochlear synaptopathy is sufficient to cause a significant gain increase that leads to significant hyperactivity in the auditory system. In addition, the ratio in which the high, medium and low spontaneous fibres are lost was determined in accordance with empirical results. This approach to modelling cochlear synaptopathy of the diverse dynamic range of AN fibres in detail in the CAP model presented here is novel.

Reduced mean firing rate, following hearing damage, triggered homeostatic changes in the DCN model which led to hyperactivity in regions of hearing damage (Figures 9 and 11). The development of hyperactivity in the DCN can almost be considered as synonymous with tinnitus. Indeed, a number of animal studies show that when hyperactivity was induced in the DCN the animals displayed behavioural signs of tinnitus with the hyperactivity occurring in regions of hearing loss [50], [51], [52], [9], [53]. Conversely in human studies, subjects with tinnitus have shown hyperactivity originating from the cochlear nucleus [5], [6]. Amplified ABRs in people with tinnitus have not always been evident [8] and consequently it is not possible to say that hyperactivity in the DCN is equivalent to the presence of tinnitus. The heterogeneity of the tinnitus population, the subjective nature of tinnitus and challenges with electrophysiological recording approaches are among the possible explanations for studies not necessarily finding elevated ABRs in all tinnitus subjects. The CAP model presented in this work could be further supported by the inclusion of tinnitus perception. The development and evaluation of a perceptual model requires the collection and analysis of relevant data from an appropriate tinnitus cohort. The addition of tinnitus sound perception is therefore a key element for future extensions of this model.

This work considered hidden hearing loss that stems from cochlear synaptopathy. Hidden hearing loss may also arise from other origins such as cochlear dead regions [54], auditory nerve demyelination [55] or reduced lateral inhibition (with or without widening of tuning curves) [56]. Future work is planned to enhance the peripheral model to account for other forms of hidden hearing loss. Moreover, it has been shown that hearing impairment can affect the VCN auditory pathway differently from the DCN auditory pathway [57]. Future work will aim to consider adaptation in both the DCN and VCN pathways in line with experimental results [5], [6]. The modular structure of the peripheral model herein easily facilitates the investigation of TCD following the creation of 


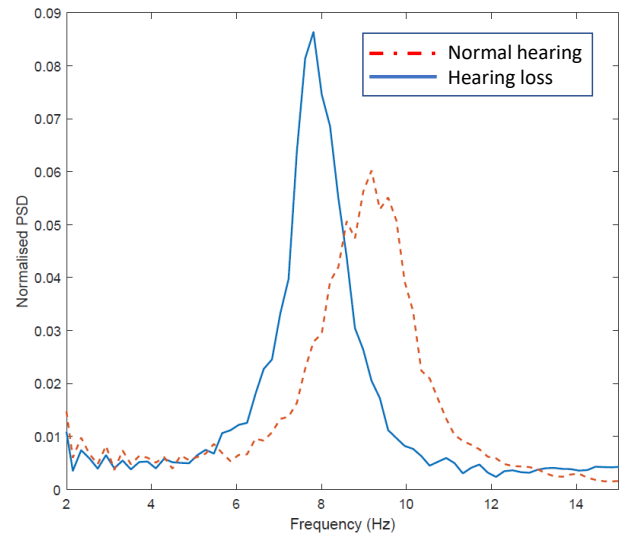

(a) Power spectrum of thalamocortical network with hearing loss and tinnitus with increased inhibition

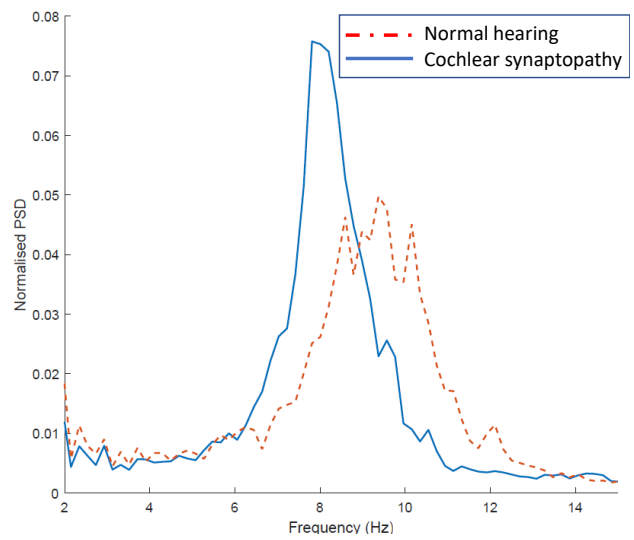

(b) Power spectrum of thalamocortical network with cochlear synaptopathy and tinnitus with increased inhibition

Fig. 14. Normalised power spectrum of tinnitus and the thalamocortical network following an increase in top-down inhibition

a richer peripheral model.

Llinas et al. [20] hypothesised that TCD in tinnitus is the result of the loss in peripheral input, due to hearing damage, which causes a reduction in the excitatory drive entering the thalamocortical network from the ascending auditory pathway. Consequently, there is excess inhibition amongst the thalamic neurons resulting in a slowing of the networks oscillatory behaviour. Our results do not align with this theory. An explanation for this is that the proposed model, in line with previous tinnitus models and experimental results [9], [5], [6], [53], [52], [51], displays an increase in the activity within the auditory brainstem following hearing loss which subsequently enters the thalamus. The key to TCD, whether it arises from bottom-up changes or top-down changes, is the overall balance between the excitatory and inhibitory signals. The results in Section III-A suggest that there is not a reduction in excitatory input to the thalamocortical network in agreement with previous models [23] and experimental findings [9], [5].

The main novelty of this paper is the combining of existing AN/DCN models with an existing thalamocortical model, and the inclusion of a biologically inspired model of cochlear synaptopathy in simulations, leading to evidence of that both a bottom-up and top-down adaptations are necessary for the perception of tinnitus. This is the first computational model to investigate TCD with tinnitus. TCD in cases of tinnitus was thought to occur due to a loss in peripheral input caused by hearing loss. However, following this development of a biologically faithful model of tinnitus related activity in the auditory periphery, characterised by enhanced DCN activity, it is noted that the peripheral input to the thalamus is not decreased as suggested in current discussions of TCD and tinnitus [20] but rather adaptive gain creates amplified peripheral activity. This is supported by animal studies showing increased activity entering the MGB of rats with induced tinnitus arising from hyperactivity from the auditory periphery [58]. If a loss in peripheral input was the sole cause of TCD in tinnitus, then hearing loss alone would produce TCD. However, it has been shown that enhanced slow wave activity is related to tinnitus rather than hearing loss [48]. A critically important finding shows that peripheral damage alone is not enough to change the behaviour of the thalamocortical network but that top-down signals are an essential component for the generation of TCD with the presence of hyperactivity in the auditory periphery. The top-down involvement was simulated by an increase in the inhibition within the thalamus. This result is supported by animal studies which have found excessive tonic inhibition is required for TCD rats with induced tinnitus [59].

The top-down involvement in the generation of TCD in people with tinnitus is thought to be representative of a deficient noise cancelling system [19], [60]. The importance of this topdown signal in generating tinnitus related oscillatory activity may also explain why a minority of people with hearing loss perceive tinnitus. If the non-adapted top-down signals exist, the tinnitus perception may not appear at a cortical level. This finding could be of great relevance when considering treatment methods for tinnitus. This paper only reveals the importance of the top-down signal and does not postulate where this signal originates. Previous models of cortical adaptation have considered possible cortical mechanisms for tinnitus generation [61], [62] as well as cortical reorganisation following hearing loss [63] without any adaptation in the auditory periphery. A number of cortical networks have been identified to be relevant to tinnitus including regions of auditory processing, memory, salience and emotion. A comprehensive review of neural imaging findings supporting this evidence is given in [64]. Without further experimental and extensive computational modelling it is not possible to determine the origins of the top-down signal and the cortical adaptation involved in the generation of this top-down signal. Future work will endeavour to find and simulate cortical adaptation, triggers for cortical adaptation and the cortical interactions involved in the top-down signal. This work would benefit from the investigation of effective connectivity; an area of limited development in the tinnitus literature.

The theoretical model of TCD describes a slowing of resting state activity from alpha to theta activity as illustrated by 
the CAP model and experimentally found in [65], [66], [67]. A caveat to accompany these findings is that the results are limited by the number of tinnitus subjects involved and the heterogeneity of the tinnitus group. Some subjects presented in [48], [21] displayed enhanced theta activity with sample size being a factor in the absence of statistical significance for further support. Other experimental studies have shown tinnitus to be related to even slower oscillatory frequencies with enhanced delta activity [48], [68], [67], [69]. In [68] it is postulated that the experimental deviation between the theoretically expected "enhanced theta activity" and the experimentally found "enhanced delta activity" could be explained by the heterogeneity of the tinnitus population, differences in frequency band boundaries and tinnitus duration. The CAP model presented here only considers changes in alpha activity to theta activity relating to TCD. Future modelling work will aim to investigate enhanced delta activity in tinnitus related TCD. This work should be complemented with empirical data and the model parameters tuned to individual subjects.

The mechanisms behind tinnitus have been postulated to be like those involved in hyperacusis [70]. Hyperacusis is a problem where people perceive sound to be abnormally loud. Approximately $85 \%$ of those with hyperacusis have tinnitus [71] while only $40 \%$ of people with tinnitus experience hyperacusis [72]. An interesting extension of this work would be to adapt the peripheral model to account for hyperacusis as investigated in [70].

\section{CONClusion}

Hearing loss is thought to be an essential component of tinnitus generation [48]. This work shows that cochlear synaptopathy is sufficient to generate tinnitus related hyperactivity in the auditory brainstem. The ascending auditory signals were previously considered to be the driving force behind TCD in people with tinnitus. However, the model presented in this paper shows that the change in input to the thalamocortical network from the auditory periphery is insufficient to cause TCD. Instead internal gain adaptation of the inhibitory connections is required to derive experimentally observable changes in the net oscillatory activity. It is likely that the bottom-up changes in the auditory system following hearing damage may instigate the changes in the top-down signal, but further work would be required to determine this. The consequence of the result suggests that tinnitus should be considered a two-way problem: firstly, peripheral damage causes changes in the bottom-up signals and, secondly, topdown signals generate oscillatory changes at a cortical level. The impact on treatment methods is such that both pathways need to be considered. Future work is needed to unify the detailed audiological research in cochlear synaptopathy and hearing loss and the neurological research that highlights the neural correlates of tinnitus. Computational modelling can then be used to investigate theories regarding the underlying mechanism that generates the observable neurological findings.

\section{ACKNOWLEDGMENT}

This work was completed under a $\mathrm{PhD}$ studentship supported by the Department of Education and Learning (DEL),
Northern Ireland, UK.

\section{REFERENCES}

[1] Berthold Langguth, Peter M Kreuzer, Tobias Kleinjung, and Dirk De Ridder. Tinnitus: causes and clinical management. The Lancet Neurology, 12(9):920-930, 2013.

[2] Cécile Nicolas-Puel, Tasnime Akbaraly, Ruth Lloyd, Claudine Berr, Alain Uziel, Guy Rebillard, and J Puel. Characteristics of tinnitus in a population of 555 patients: specificities of tinnitus induced by noise trauma. International Tinnitus Journal, 12(1):64, 2006.

[3] Adam C Furman, Sharon G Kujawa, and M Charles Liberman. Noiseinduced cochlear neuropathy is selective for fibers with low spontaneous rates. Journal of neurophysiology, 110(3):577-586, 2013.

[4] Marmel, Frédéric and Rodríguez-Mendoza, Medardo A and LopezPoveda, Enrique A. Stochastic undersampling steepens auditory threshold/duration functions: implications for understanding auditory deafferentation and aging. Frontiers in aging neuroscience, 7:1-13, 2015.

[5] Roland Schaette and David McAlpine. Tinnitus with a normal audiogram: physiological evidence for hidden hearing loss and computational model. The Journal of Neuroscience, 31(38):13452-13457, 2011.

[6] Jianwen Wendy Gu, Barbara S Herrmann, Robert A Levine, and Jennifer R Melcher. Brainstem auditory evoked potentials suggest a role for the ventral cochlear nucleus in tinnitus. Journal of the Association for Research in Otolaryngology, 13(6):819-833, 2012.

[7] Liberman, M Charles and Epstein, Michael J and Cleveland, Sandra S and Wang, Haobing and Maison, Stéphane F. Toward a differential diagnosis of hidden hearing loss in humans. PLoS One, 11(9):e0162726, 2016.

[8] Hannah Guest, Kevin J Munro, Garreth Prendergast, Simon Howe, and Christopher J Plack. Tinnitus with a normal audiogram: Relation to noise exposure but no evidence for cochlear synaptopathy. Hearing research, 344:265-274, 2017.

[9] James A Kaltenbach, Mark A Zacharek, Jinsheng Zhang, and Sarah Frederick. Activity in the dorsal cochlear nucleus of hamsters previously tested for tinnitus following intense tone exposure. Neuroscience letters, 355(1-2):121-125, 2004.

[10] Seth D Koehler and Susan E Shore. Stimulus timing-dependent plasticity in dorsal cochlear nucleus is altered in tinnitus. The Journal of Neuroscience, 33(50):19647-19656, 2013.

[11] Roland Schaette and Richard Kempter. Predicting tinnitus pitch from patients' audiograms with a computational model for the development of neuronal hyperactivity. Journal of neurophysiology, 101(6):3042-3052, 2009.

[12] Helga M Kehrle, Ronaldo C Granjeiro, André LL Sampaio, Roberta Bezerra, Vanessa F Almeida, and Carlos A Oliveira. Comparison of auditory brainstem response results in normal-hearing patients with and without tinnitus. Archives of Otolaryngology-Head \& Neck Surgery, 134(6):647-651, 2008.

[13] Lara Li Hesse, Warren Bakay, Hui-Ching Ong, Lucy Anderson, Jonathan Ashmore, David McAlpine, Jennifer Linden, and Roland Schaette. Nonmonotonic relation between noise exposure severity and neuronal hyperactivity in the auditory midbrain. Frontiers in neurology, 7, 2016.

[14] Golbarg Mehraei, Ann E Hickox, Hari M Bharadwaj, Hannah Goldberg, Sarah Verhulst, M Charles Liberman, and Barbara G Shinn-Cunningham. Auditory brainstem response latency in noise as a marker of cochlear synaptopathy. The Journal of Neuroscience, 36(13):3755-3764, 2016.

[15] BT Paul, IC Bruce, and LE Roberts. Amplitude modulation encoding deficits suggest hidden hearing loss in individuals with tinnitus and normal audiograms. Peripheral and Central Factors in Tinnitus, page 47.

[16] Roland Schaette, Charlotte Turtle, and Kevin J Munro. Reversible induction of phantom auditory sensations through simulated unilateral hearing loss. PLoS One, 7(6):e35238, 2012.

[17] Kevin J Munro and Jennifer Blount. Adaptive plasticity in brainstem of adult listeners following earplug-induced deprivation. The Journal of the Acoustical Society of America, 126(2):568-571, 2009.

[18] Gastone G. Celesia. Chapter 7 - Brainstem auditory evoked responses in Disorders of Peripheral and Central Auditory Processing (Handbook of Clinical Neurophysiology). Elsevier, 2013(10):1567-4231, 2013.

[19] Dirk De Ridder, Sven Vanneste, Berthold Langguth, and Rodolfo Llinas. Thalamocortical dysrhythmia: a theoretical update in tinnitus. Frontiers in neurology, 6, 2015.

[20] Rodolfo Llinás, Francisco J Urbano, Elena Leznik, Rey R Ramírez, and Hein JF van Marle. Rhythmic and dysrhythmic thalamocortical dynamics: Gaba systems and the edge effect. Trends in neurosciences, 28(6):325$333,2005$. 
[21] Nathan Weisz, Stephan Moratti, Marcus Meinzer, Katalin Dohrmann, and Thomas Elbert. Tinnitus perception and distress is related to abnormal spontaneous brain activity as measured by magnetoencephalography. PLoS Med, 2(6):e153, 2005.

[22] Nathan Weisz, Simona Müller, Winfried Schlee, Katalin Dohrmann, Thomas Hartmann, and Thomas Elbert. The neural code of auditory phantom perception. The Journal of Neuroscience, 27(6):1479-1484, 2007.

[23] Roland Schaette and Richard Kempter. Development of hyperactivity after hearing loss in a computational model of the dorsal cochlear nucleus depends on neuron response type. Hearing research, 240(1):57-72, 2008.

[24] Roland Schaette and Richard Kempter. Development of tinnitus-related neuronal hyperactivity through homeostatic plasticity after hearing loss: a computational model. European Journal of Neuroscience, 23(11):31243138, 2006.

[25] Carney, Laurel H A model for the responses of low-frequency auditorynerve fibers in cat. The Journal of the Acoustical Society of America, 93(1):401-417, 1993.

[26] Zhang, Xuedong and Heinz, Michael G and Bruce, Ian C and Carney, Laurel $\mathrm{H}$ A phenomenological model for the responses of auditory-nerve fibers: I. Nonlinear tuning with compression and suppression. The Journal of the Acoustical Society of America, 109(2):648-670, 2001.

[27] Bruce, Ian C and Sachs, Murray B and Young, Eric D An auditoryperiphery model of the effects of acoustic trauma on auditory nerve responses. The Journal of the Acoustical Society of America, 113(1):369388, 2003.

[28] Zilany, Muhammad SA and Bruce, Ian C Modeling auditory-nerve responses for high sound pressure levels in the normal and impaired auditory periphery. The Journal of the Acoustical Society of America, 120(3):1446-1466, 2006.

[29] Zilany, Muhammad SA and Bruce, Ian C Representation of the vowel/ $/$ /in normal and impaired auditory nerve fibers: model predictions of responses in cats. The Journal of the Acoustical Society of America, 122(1):402-417, 2007.

[30] Muhammad SA Zilany, Ian C Bruce, Paul C Nelson, and Laurel H Carney. A phenomenological model of the synapse between the inner hair cell and auditory nerve: long-term adaptation with power-law dynamics. The Journal of the Acoustical Society of America, 126(5):2390-2412, 2009.

[31] Muhammad SA Zilany and Laurel H Carney. Power-law dynamics in an auditory-nerve model can account for neural adaptation to sound-level statistics. Journal of Neuroscience, 30(31):10380-10390, 2010.

[32] Muhammad SA Zilany, Ian C Bruce, and Laurel H Carney. Updated parameters and expanded simulation options for a model of the auditory periphery. The Journal of the Acoustical Society of America, 135(1):283286, 2014.

[33] Donald D Greenwood. A cochlear frequency-position function for several species-29 years later. The Journal of the Acoustical Society of America, 87(6):2592-2605, 1990.

[34] M Charles Liberman. Auditory-nerve response from cats raised in a low-noise chamber. The Journal of the Acoustical Society of America, 63(2):442-455, 1978

[35] Kenneth E Hancock and Herbert F Voigt. Wideband inhibition of dorsal cochlear nucleus type iv units in cat: a computational model. Annals of biomedical engineering, 27(1):73-87, 1999.

[36] J Henning Proske, Daniel Jeanmonod, and Paul FMJ Verschure. A computational model of thalamocortical dysrhythmia. European Journal of Neuroscience, 33(7):1281-1290, 2011.

[37] Dale Purves, George J Augustine, David Fitzpatrick, Lawrence C Katz, Anthony-Samuel LaMantia, James O McNamara, and S Mark Williams. The Auditory Thalamus. Sinauer Associates, 2001.

[38] NF Manzoor, Y Gao, F Licari, and JA Kaltenbach. Comparison and contrast of noise-induced hyperactivity in the dorsal cochlear nucleus and inferior colliculus. Hearing research, 295:114-123, 2013.

[39] NF Manzoor, Frank G Licari, Michael Klapchar, RL Elkin, Yuan Gao, G Chen, and JA Kaltenbach. Noise-induced hyperactivity in the inferior colliculus: its relationship with hyperactivity in the dorsal cochlear nucleus. Journal of neurophysiology, 108(4):976-988, 2012.

[40] Basabdatta Sen-Bhattacharya, Neslihan Serap-Sengor, Yuksel Cakir, Liam Maguire, and Damien Coyle. Spectral and non-linear analysis of thalamocortical neural mass model oscillatory dynamics. In Advanced Computational Approaches to Biomedical Engineering, pages 87-112. Springer, 2014.

[41] Gregory D Smith, Charles L Cox, S Murray Sherman, and John Rinzel. Fourier analysis of sinusoidally driven thalamocortical relay neurons and a minimal integrate-and-fire-or-burst model. Journal of Neurophysiology, 83(1):588-610, 2000.
[42] Alain Destexhe and Terrence J Sejnowski. The initiation of bursts in thalamic neurons and the cortical control of thalamic sensitivity. Philosophical Transactions of the Royal Society B: Biological Sciences, 357(1428):1649-1657, 2002.

[43] M Charles Liberman, Leslie W Dodds, and Sarah Pierce. Afferent and efferent innervation of the cat cochlea: quantitative analysis with light and electron microscopy. Journal of Comparative Neurology, 301(3):443-460, 1990.

[44] Sharon G Kujawa and M Charles Liberman. Adding insult to injury: cochlear nerve degeneration after "temporary" noise-induced hearing loss. The Journal of Neuroscience, 29(45):14077-14085, 2009.

[45] https://www.urmc.rochester.edu/labs/CarLab/publications/auditorymodels.aspx

[46] Moazami-Goudarzi, M., Michels, L., Weisz, N., and Jeanmonod, D. (2010). Temporo-insular enhancement of eeg low and high frequencies in patients with chronic tinnitus. qeeg study of chronic tinnitus patients. BMC neuroscience, 11(1):40.

[47] Vanneste, S., Song, J.-J., and De Ridder, D. (2013a). Tinnitus and musical hallucinosis: the same but more. Neuroimage, 82:373-383.

[48] Peyman Adjamian, Magdalena Sereda, Oliver Zobay, Deborah A Hall, and Alan R Palmer. Neuromagnetic indicators of tinnitus and tinnitus masking in patients with and without hearing loss. Journal of the Association for Research in Otolaryngology, 13(5):715-731, 2012.

[49] Kahlbrock, N. and Weisz, N. (2008). Transient reduction of tinnitus intensity is marked by concomitant reductions of delta band power. $B M C$ biology, 6(1):4.

[50] JS Zhang, JA Kaltenbach, DA Godfrey, and J Wang. Origin of hyperactivity in the hamster dorsal cochlear nucleus following intense sound exposure. Journal of neuroscience research, 84(4):819-831, 2006.

[51] TJ Brozoski, CA Bauer, and DM Caspary. Elevated fusiform cell activity in the dorsal cochlear nucleus of chinchillas with psychophysical evidence of tinnitus. Journal of Neuroscience, 22(6):2383-2390, 2002.

[52] Henry E Heffner and Ian A Harrington. Tinnitus in hamsters following exposure to intense sound. Hearing research, 170(1-2):83-95, 2002.

[53] James A Kaltenbach and Jinsheng Zhang. Intense sound-induced plasticity in the dorsal cochlear nucleus of rats: evidence for cholinergic receptor upregulation. Hearing research, 226(1-2):232-243, 2007.

[54] Moore, BCJ and Huss, M and Vickers, DA and Glasberg, BR and Alcántara, JI. A test for the diagnosis of dead regions in the cochlea. British journal of audiology, 34(4):205-224, 2000.

[55] Wan, Guoqiang and Corfas, Gabriel. Transient auditory nerve demyelination as a new mechanism for hidden hearing loss. Nature Communications, 8:1-13,2017.

[56] Takesian, Anne E and Kotak, Vibhakar C and Sanes, Dan H. Developmental hearing loss disrupts synaptic inhibition: implications for auditory processing. Future Neurology, 4(3):331-349,2009.

[57] Cai, Shanqing and Ma, Wei-Li D and Young, Eric D. Encoding intensity in ventral cochlear nucleus following acoustic trauma: implications for loudness recruitment. Journal of the Association for Research in Otolaryngology, 10(1):5-22,2009.

[58] Bopanna I Kalappa, Thomas J Brozoski, Jeremy G Turner, and Donald M Caspary. Single unit hyperactivity and bursting in the auditory thalamus of awake rats directly correlates with behavioural evidence of tinnitus. The Journal of physiology, 592(22):5065-5078, 2014.

[59] Evgeny A Sametsky, Jeremy G Turner, Deb Larsen, Lynne Ling, and Donald M Caspary. Enhanced gabaa-mediated tonic inhibition in auditory thalamus of rats with behavioral evidence of tinnitus. Journal of Neuroscience, 35(25):9369-9380, 2015.

[60] Josef P Rauschecker, Amber M Leaver, and Mark Mühlau. Tuning out the noise: limbic-auditory interactions in tinnitus. Neuron, 66(6):819-826, 2010.

[61] Dominguez, Melissa and Becker, Suzanna and Bruce, Ian and Read, Heather. A spiking neuron model of cortical correlates of sensorineural hearing loss: spontaneous firing, synchrony, and tinnitus. Neural computation, 18(12):2942-2958, 2006.

[62] Chrostowski, Michael and Yang, Le and Wilson, Hugh R and Bruce, Ian C and Becker, Suzanna. Can homeostatic plasticity in deafferented primary auditory cortex lead to travelling waves of excitation? Journal of computational neuroscience, 30(2):279-299, 2011.

[63] Tomková, Markéta and Tomek, Jakub and Novák, Ondřej and Zelenka, Ondřej and Syka, Josef and Brom, Cyril. Formation and disruption of tonotopy in a large-scale model of the auditory cortex. Journal of computational neuroscience, 39(2):131-153, 2015.

[64] Ana Belén Elgoyhen, Berthold Langguth, Dirk De Ridder, and Sven Vanneste. Tinnitus: perspectives from human neuroimaging. Nature Reviews Neuroscience, 16(10):632, 2015. 
[65] Rodolfo R Llinás, Urs Ribary, Daniel Jeanmonod, Eugene Kronberg, and Partha P Mitra. Thalamocortical dysrhythmia: a neurological and neuropsychiatric syndrome characterized by magnetoencephalography. Proceedings of the National Academy of Sciences, 96(26):15222-15227, 1999.

[66] Dirk De Ridder, Elsa van der Loo, Sven Vanneste, Steffen Gais, Mark Plazier, Silvia Kovacs, Stefan Sunaert, Tomas Menovsky, and Paul Van de Heyning. Theta-gamma dysrhythmia and auditory phantom perception: case report. Journal of neurosurgery, 114(4):912-921, 2011.

[67] Rey René Ramírez, Brian Harris Kopell, Christopher R Butson, Wolfgang Gaggl, David R Friedland, and Sylvain Baillet. Neuromagnetic source imaging of abnormal spontaneous activity in tinnitus patient modulated by electrical cortical stimulation. In Engineering in Medicine and Biology Society, 2009. EMBC 2009. Annual International Conference of the IEEE, pages 1940-1944. IEEE, 2009.

[68] Ilya Adamchic, Timea Toth, Christian Hauptmann, and Peter Alexander Tass. Reversing pathologically increased eeg power by acoustic coordinated reset neuromodulation. Human brain mapping, 35(5):2099-2118, 2014.

[69] Sven Vanneste, Paul Van de Heyning, and Dirk De Ridder. The neural network of phantom sound changes over time: a comparison between recent-onset and chronic tinnitus patients. European Journal of Neuroscience, 34(5):718-731, 2011.

[70] Peter U Diehl and Roland Schaette. Abnormal auditory gain in hyperacusis: investigation with a computational model. Frontiers in neurology, 6, 2015.

[71] Jacqueline Sheldrake, Peter U Diehl, and Roland Schaette. Audiometric characteristics of hyperacusis patients. Frontiers in neurology, 6, 2015.

[72] A Fabijanska, M Rogowski, G Bartnik, and H Skarzynski. Epidemiology of tinnitus and hyperacusis in poland. In Proceedings of the sixth international tinnitus seminar, pages 569-571. The Tinnitus and Hyperacusis Centre Cambridge, 1999 